\title{
LOWERING ORDERS OF DERIVATIVES IN NON-LINEAR RESIDUAL GENERATION
}

\author{
Jan Åslund ${ }^{1}$, Erik Frisk $^{1}$ \\ ${ }^{1}$ Department of Electrical Engineering, Linköpings universitet, 58183 \\ Linköping, Sweden, \{jaasl, frisk\}eisy.liu.se
}

\begin{abstract}
Consistency relations are often used to design residual generators based on nonlinear process models. A main difficulty is that they generally include time differentiated versions of known signals which are difficult to estimate in a noisy environment. The main results of this paper show how to lower, or if possible avoid, the need to estimate derivatives of known signals in order to compute a residual. This is achieved by rewriting the problem into an integrability problem using state-space realization theory. An attractive feature of the approach is that general differential algebraic system descriptions can be handled in the same way as for example ordinary differential equations and also that stability of the residual generator is always guaranteed. Copyright ${ }^{\complement} 2005$ IFAC.
\end{abstract}

Keywords: Fault diagnosis, Parity relations, Analytical redundancy, Differential geometry, Non-linear systems

\section{INTRODUCTION}

The objective of fault diagnosis is to detect and isolate any faults acting on a process. In many methods, e.g. structured residuals (Gertler, 1991), the concept of residuals plays a central role. A residual is a signal that ideally is zero when there is no fault acting on the process and that deviates from zero when a fault occurs. Commonly, a set of residuals is used, where different residuals are sensitive to different subsets of faults and in this way isolation between faults is possible. Residual generation for fault diagnosis based on non-linear dynamical models has in the control community mainly been performed in two ways, either via non-linear state observation or via non-linear consistency relations. Both approaches have their own set of advantages and disadvantages and a notable contribution using observers is (Persis and Isidori, 2001). If non-linear consistency relations are used for designing residual generators to fit in a structured residuals framework, the decoupling problem is transformed

\footnotetext{
1 Funding support from the Swedish Foundation for Strategic Research and the Center for Industrial Information Technology are gratefully acknowledged.
}

into an elimination problem. For a general description of a method to derive consistency relations, see (Staroswiecki and Comtet-Varga, 2001).

An attractive property of such approaches is for example that the systems considered need not to be on e.g. control-affine form but can also include algebraic constraints. Consistency relations have proven beneficial in the linear case as a basis for residual generators, but when generalizing to the non-linear problem some additional difficulties arise. A main difficulty is that the consistency relation generally include time differentiated versions of known signals and these are not normally known. For linear systems, this problem is easily circumvented. Adding residual generator dynamics of high enough order and finding a statespace realization using linear realization theory makes it possible to compute a residual without the need to approximate any derivatives (Frisk and Nyberg, 2001). The aim of this work is to generalize this procedure also for non-linear systems by transforming the problem into an integrability problem. This work is a first step toward a general solution and lowers the order of derivatives of as many variables as possible one step. 
A related problem has been studied in detail in (Delaleau and Respondek, 1995) where the orders of derivatives in generalized state-space descriptions are lowered.

\section{PROBLEM FORMULATION}

An important concept when designing residual generators is consistency relations which can be defined as:

Definition 1. (Consistency Relation). Let $u(t)$ be a vector of all known signals. The scalar expression $c(u)$ is a consistency relation if for all $u$ consistent with the fault free model it holds that

$$
c(u, \dot{u}, \ddot{u}, \ldots)=0
$$

The order of a consistency relation is defined as the highest order of a derivative of a known signal in the relation. Note that $u$ includes both measurements and known control signals. Therefore, testing if $c$ equal 0 is a test on consistency between measured data and the equations/assumptions used when deriving $c$. A fault is detectable in (1) if the relation does not hold in case the fault occurs.

Example 1. An example of how a consistency relation can be obtained is illustrated in this example. Consider the following model from (Persis and Isidori, 2001)

$$
\begin{aligned}
& \dot{x}_{1}(t)=x_{2}(t) \\
& \dot{x}_{2}(t)=x_{1}(t) x_{4}^{2}(t)-\theta_{1} \frac{1}{x_{1}^{2}(t)}+\theta_{2} u_{1}(t)+d(t) \\
& \dot{x}_{3}(t)=x_{4}(t) \\
& \dot{x}_{4}(t)=-2 \frac{x_{2}(t) x_{4}(t)}{x_{1}(t)}+\theta_{2} \frac{u_{2}(t)}{x_{1}(t)}+\theta_{2} \frac{f(t)}{x_{1}(t)} \\
& y_{1}(t)=x_{1}(t), y_{2}(t)=x_{3}(t), y_{3}(t)=x_{4}(t)
\end{aligned}
$$

where $y_{i}$ are known measurements, $u_{i}$ known inputs, $\theta_{i}$ known constants, $d$ unknown disturbance, and $f$ the fault we want to detect.

A relation, not sensitive to the disturbance $d$, can be derived by direct substitution of $y_{i}$ and $x_{2}$ into the fourth equation

$$
y_{1} \dot{y}_{3}+2 \dot{y}_{1} y_{3}-\theta_{2} u_{2}=\theta_{2} f
$$

Thus, the left hand side $c=y_{1} \dot{y}_{3}+2 \dot{y}_{1} y_{3}-\theta_{2} u_{2}$ is a consistency relation since $c=0$ in the fault-free case, i.e. when $f=0$. The right hand side $s=\theta_{2} f$ is the fault sensitivity.

The most straightforward way to generate a residual using a consistency relation would be

$$
r=c(u, \dot{u}, \ldots)
$$

To compute a residual according to (2) it would be necessary to estimate the time derivatives which may be difficult, especially for higher order derivatives, in a noisy environment. The main topic of this paper is to describe ways to lower the order of derivatives needed to be estimated.
If $c(u, \dot{u}, \ldots)$ was linear we could add any stable linear residual generator dynamics of order high enough, at least as large as the highest input derivative:

$$
r^{(n)}+a_{n} r^{(n-1)}+\cdots+a_{1} r=c(u, \dot{u}, \ldots) .
$$

For example, if $u+\dot{u}+\ddot{u}=0$ is a consistency relation, adding second order residual generator dynamics and writing the residual generator on operator form give

$$
r(t)=\frac{p^{2}+p+1}{p^{2}+a_{2} p+a_{1}} u(t)
$$

It is clear that this can easily be written on explicit state-space form and that the residual $r$ can be computed without the need to estimate any derivatives. This is the approach that in the following sections will be mimicked for the non-linear case. Unfortunately, but hardly surprising, is that the realization step that was immediate in the linear case is not direct in the non-linear case.

A generalization, for the case of a first order consistency relation, of the above approach for the nonlinear case would be to find residual generator dynamics $h$ and a transformation $\Psi$ such that

$$
\dot{r}+h(r, u)=\Psi(c(\dot{u}, u), u)
$$

can be realized on state-space form with no differentiated inputs. The functions $h$ and $\Psi$ must quite naturally satisfy the constraints that $r=0$ is a stable locus of the differential equation

$$
\dot{r}+h(r, u)=0
$$

and that $\Psi(0, u)=0$ for all $u$. This means that the residual generator is stable and that if $c(u)$ is a consistency relation so is $\Psi(c(\dot{u}, u), u)$. Note that if there exists $u$ such that $\Psi(c, u)=0$ for $c \neq 0$, the transformation of the original consistency relation may reduce the detectability performance of the residual compared to the detectability of the original consistency relation.

Now, an example will be presented that shows how $h$ and $\Psi$ can be chosen in a specific case. General methodology will be presented in subsequent sections. Example 2. Consider the same non-linear consistency relation as in Example 1

$$
c(\dot{u}, u)=u_{2} \dot{u}_{1}+2 u_{1} \dot{u}_{2}+\theta u_{3}
$$

where $u$ is the vector of known signals as in (1). It is straightforward to verify that it is not possible to put $\dot{r}+\beta r=c(\dot{u}, u)$ on explicit state-space form and a transformation of $c$ is necessary. Now, choose $\Psi(c(\dot{u}, u), u)=u_{2} c(\dot{u}, u)$, to obtain a new consistency relation

$$
\begin{aligned}
\Psi(c(\dot{u}, u), u) & =u_{2}^{2} \dot{u}_{1}+2 u_{1} u_{2} \dot{u}_{2}+\theta u_{3} u_{2} \\
& =\frac{d}{d t}\left(u_{1} u_{2}^{2}\right)+\theta u_{3} u_{2}
\end{aligned}
$$

which is linear after a change of variables. Then the linear approach applies to

$$
\dot{r}+h(r, u)=\Psi(c(\dot{u}, u), u)
$$


with linear residual generator dynamics, i.e. $h(r, u)=$ $\beta r$, and the corresponding state-space realization is given by

$$
\begin{aligned}
\dot{w} & =-\beta w-\beta u_{1} u_{2}^{2}+\theta u_{3} u_{2} \\
r & =w+u_{1} u_{2}^{2}
\end{aligned}
$$

Now, the main objectives of the paper are summarized.

Problem formulation: Given a set of consistency relations, the objective is to investigate possible transformations of these relations to obtain a new set of consistency relations that can be used to compute residuals where the order of the derivatives have been lowered by one.

The goal is to put the residual generator on state-space form using established realization theory. It is important to note that in the residual generation problem, the residual is not uniquely defined, the only requirement is that the residual is sensitive to a predefined set of faults. This introduces some extra design freedom not available in standard realization procedures. Utilization of this extra freedom is instrumental in the proposed solution.

First we consider consistency relations of order one and derive conditions for when it is possible to remove all derivatives. For cases where all derivatives can not be removed, partial solutions may exist where the number of differentiated inputs in the residual generator is lowered. These possibilities are described further in Section 7 where it is also described how the methodology can be used on higher order consistency relations to lower the order of the derivatives one step.

\section{INTRODUCING RESIDUAL GENERATOR DYNAMICS}

The objective of this section is to investigate when there exists residual generator dynamics $h(r, u)$ such that

$$
\dot{r}+h(r, u)=c(\dot{u}, u)
$$

can be transformed into a stable state-space realization with no input derivatives. It is assumed that the function $c$ is differentiable. The results presented below form a foundation for a more general approach presented in the next section where also the transformation $\Psi$ is also considered.

First, assume that the consistency relation can be written on the form

$$
c(\dot{u}, u)=\sum_{i=1}^{n} g_{i}(u) \dot{u}_{i}+v(u)
$$

and that the functions $g_{i}$ are differentiable. If the vector field

$$
g(u)=\left(g_{1}(u), \ldots, g_{n}(u)\right)
$$

has a potential, i.e.

$$
g_{i}=\frac{\partial \lambda}{\partial u_{i}}
$$

for some function $\lambda(u)$ then it holds that

$$
\frac{d}{d t} \lambda(u)=\sum_{i=1}^{n} g_{i}(u) \dot{u}_{i}
$$

In this case we can choose linear residual generator dynamics

$$
h(r, u)=\beta r, \quad \beta>0
$$

and rewrite (4) into a linear problem

$$
\dot{r}+\beta r=\frac{d}{d t} \lambda(u)+v(u)
$$

which can easily be written on state-space form. According to Poincare's Lemma there exists a potential $\lambda$ if and only if the vector field $g$ fulfills the condition

$$
\frac{\partial g_{i}}{\partial u_{j}}-\frac{\partial g_{j}}{\partial u_{i}}=0
$$

for all $i, j$. Hence, condition (7) is sufficient for the existence of residual generator dynamics that makes it possible to write the residual generator on statespace form. Also, it is always possible to choose linear dynamics which makes it easy to ensure stability of the filter.

The following Lemma shows that conditions (5) and (7) are in fact also necessary. The proof is omitted and is a slight adaptation of the proofs in (Delaleau and Respondek, 1995).

Lemma 1. Let $c(\dot{u}, u)$ be a consistency relation of order 1 . There exists residual generator dynamics $h(r, u)$ and a change of coordinates such that (4) is transformed into a state-space realization with no input derivatives if and only if $c(\dot{u}, u)$ can be written on the form (5) and there exists a potential for the vector field $g$.

\section{USING TRANSFORMED CONSISTENCY RELATIONS}

The previous section introduced residual generator dynamics to remove derivatives in the computational form. Now the approach will be extended by allowing a transformation of the consistency relation. The objective of this section is to determine when there exists residual generator dynamics $h$ and a transformation $\Psi$ such that (3) can be transformed into a state-space realization with no input derivatives. The difference between this problem and the standard problem of non-linear system realization is that in this case, we have the freedom to choose the functions $h$ and $\Psi$. The objective is to choose these functions so that realization is possible.

According to Lemma 1 it is necessary that $\Psi(c(\dot{u}, u), u)$ can be written on the form (5). If the original consistency relation $c(\dot{u}, u)$ can be written on this form, then 
the only possible transformation is to multiply by a function $\alpha(u)$ as in Example 2. Therefore the procedure to find the transformation $\Psi$ can be divided into two steps. First, if it is possible, construct a transformation so that a consistency relation on the form (5) is obtained. Then determine if there exists an integrating factor $\alpha(u)$.

The first step, i.e. construction of a transformation $\Psi_{1}$ such that $\Psi_{1}(c(\dot{u}, u), u)$ is on form (5), is usually either trivial or impossible. Thus, this is not pursued further here and it is assumed that this step has been successful. In the previous section it was shown that it is no restriction to only consider linear residual generator dynamics. The remaining problem is now to state necessary and sufficient conditions for the existence of an integrating factor $\alpha$ so that the equation

$$
\dot{r}+\beta r=\alpha(u) c(\dot{u}, u)
$$

can be transformed into a state-space realization with no input derivatives.

Looking back at the discussion in the previous section, the difference is that vector field $g$ has now been replaced by $\alpha g$. This implies that the problem here is equivalent to the existence of a function $\alpha$ such that the vector field $\alpha g$ has a potential. Below is a key result giving necessary and sufficient conditions. It is important to note that in contrast to the global result in the previous section, the result below is local.

Theorem 1. Let $c(\dot{u}, u)$ be a consistency relation of order 1 . There exists, in a neighborhood of a point $u_{0}$, an integrating factor $\alpha(u) \not \equiv 0$ and a change of coordinates such that (8) is transformed into a statespace realization with no input derivatives if and only if $c(\dot{u}, u)$ can be written on the form (5) where the functions $g_{i}$ satisfy the condition

$$
\begin{aligned}
g_{i}\left(\frac{\partial g_{k}}{\partial u_{j}}-\frac{\partial g_{j}}{\partial u_{k}}\right)+ & g_{j}\left(\frac{\partial g_{i}}{\partial u_{k}}-\frac{\partial g_{k}}{\partial u_{i}}\right) \\
& +g_{k}\left(\frac{\partial g_{j}}{\partial u_{i}}-\frac{\partial g_{i}}{\partial u_{j}}\right)=0
\end{aligned}
$$

for all $i, j, k$ and $g \neq 0$ in a neighborhood of $u_{0}$.

PROOF. It follows from Lemma 1 and the discussion above that the problem here is equivalent to the existence of an $\alpha$ such that $\alpha g$ has a potential. A necessary and sufficient condition is given by the Frobenius theorem presented in the next section. Condition (9) is a nontrivial reformulation of condition (14). See (Choquet-Bruhat et al., 1982) for details.

Remark 1. It is straightforward to verify that in case of two differentiated inputs, the condition in the theorem is always fulfilled.

Remark 2. In three dimensions the conditions (7) and (9) can be written $\operatorname{curl}(g)=0$ and $\operatorname{curl}(g) \cdot g=0$ respectively.

\section{COMBINE CONSISTENCY RELATIONS}

There are cases where the conditions in Theorem 1 are not fulfilled. However, for a given system there often exists more than one consistency relation. This section develops an extension to the previous section and more than one relation is used in the design. Such an extension relaxes the restrictiveness of condition (9) in Theorem 1. A detailed description of the mathematical background used in this section can be found in e.g. (Isidori, 1995). In this section, results from differential geometry is used and in this framework, the vector fields $g$ in (5) are covector fields.

Given a set of consistency relations $c_{i}(\dot{u}, u)=0$, $i=1, \ldots, r$, the objective is to combine the set of consistency relations into one such that the methodology from the previous section can be applied. Note that, the discussion below will only include sufficient conditions for existence of such a combination of consistency relations. No necessary conditions are included.

Now, following the discussion regarding possible transformations in Section 4 , it is from now on assumed that the class of transformations used to combine the consistency relations is restricted to:

$$
c(\dot{u}, u)=\alpha_{1}(u) c_{1}(\dot{u}, u)+\ldots+\alpha_{r}(u) c_{r}(\dot{u}, u)=0
$$

It is, as in the previous section, assumed that the functions $c_{i}(\dot{u}, u)$ can be written on the form (5) and the corresponding covector fields are denoted by $g_{i}$. It follows that $c(\dot{u}, u)$ can be written on the same form (5) and the corresponding covector field $g$ is given by

$$
g(u)=\alpha_{1}(u) g_{1}(u)+\ldots+\alpha_{r}(u) g_{r}(u)
$$

In the present case, the question at issue is whether or not there exist a potential $\lambda$ and coefficient functions $\alpha_{1}, \ldots, \alpha_{r}$ such that

$$
d \lambda(u)=\alpha_{1}(u) g_{1}(u)+\ldots+\alpha_{r}(u) g_{r}(u)
$$

Let the codistribution $\Omega$ be defined by

$$
\Omega(u)=\operatorname{span}\left\{g_{1}(u), \ldots, g_{r}(u)\right\}
$$

Equation (11) can then be written as

$$
d \lambda \in \Omega
$$

It is assumed that $\Omega$ is non-singular in a neighborhood $\mathcal{U}$ of a point $u_{0}$, i.e. the dimension of $\Omega(u)$ is constant in $\mathcal{U}$. Without loss of generality, the covector fields $g_{i}$ are assumed to be independent, i.e. the dimension of $\Omega$ is $r$. Frobenius theorem gives a necessary and sufficient condition for the local existence of a set of functions $\lambda_{1}(u), \ldots, \lambda_{r}(u)$ such that

$$
\operatorname{span}\left\{d \lambda_{1}(u), \ldots, d \lambda_{r}(u)\right\}=\Omega(u)
$$

in a neighborhood of $u_{0}$. In this case, any of the functions $\lambda_{1}, \ldots, \lambda_{r}$ fulfills (12). Equation (12) is equivalent to

$$
d \lambda \Omega^{\perp}=0
$$

where $\Omega^{\perp}$ is the distribution that for every point assigns the subspace orthogonal to $\Omega$. The condition in 
Frobenius theorem, for a spanning solution (13), is that the distribution $\Omega^{\perp}$ has to be involutive, which means that the following condition has to be fulfilled:

$$
\tau_{1} \in \Omega^{\perp}, \tau_{2} \in \Omega^{\perp} \Longrightarrow\left[\tau_{1}, \tau_{2}\right] \in \Omega^{\perp}
$$

where $\left[\tau_{1}, \tau_{2}\right]$ denotes the Lie bracket of the vector fields $\tau_{1}$ and $\tau_{2}$. Note that this condition is always fulfilled if the dimension of $\Omega^{\perp}$ is equal to one, i.e the dimension of $\Omega$ is $n-1$. This is an extension of Remark 1 to dimensions higher than two. Practically, this means that if there is one more differentiated input than consistency relations, that can be written on form (5), the methodology will succeed.

For our purpose it is not necessary to find a set of potentials whose gradients span the codistribution $\Omega$. Even if condition (14) is not fulfilled, it may still be possible construct a smaller set of functions $\left\{\lambda_{1}, \ldots, \lambda_{q}\right\}$ that fulfill condition (12) and span a $q$ dimensional subspace that is contained in $\Omega$ at every point. The largest codistribution spanned by gradients is obtained as follows. First, construct the involutive closure inv $\left(\Omega^{\perp}\right)$ of $\Omega^{\perp}$, i.e. the smallest involutive distribution that contains $\Omega^{\perp}$. Then the annihilator (inv $\left.\left(\Omega^{\perp}\right)\right)^{\perp}$ of this distribution is the sought codistribution. If this codistribution is the zero codistribution then no solution exists. The involutivity condition in Frobenius theorem is fulfilled by construction and there exist functions $\left\{\lambda_{1}, \ldots, \lambda_{q}\right\}$ such that

$$
\operatorname{span}\left\{d \lambda_{1}, \ldots, d \lambda_{q}\right\}=\left(\operatorname{inv}\left(\Omega^{\perp}\right)\right)^{\perp} \subset \Omega
$$

Example 3. Consider two consistency relations:

$$
\begin{aligned}
& c_{1}(u, \dot{u})=u_{2} \dot{u}_{1}+\dot{u}_{2}+\dot{u}_{3} \\
& c_{2}(u, \dot{u})=u_{2} \dot{u}_{1}+A u_{1} \dot{u}_{2}
\end{aligned}
$$

where $A$ is a known constant. Assume the objective is to detect any fault that influence either $c_{1}$ or $c_{2}$. It is clear that both is on form (5) with the corresponding covector fields $g_{1}=\left[\begin{array}{lll}u_{2} & 1 & 1\end{array}\right], g_{2}=\left[\begin{array}{lll}u_{2} & A u_{1} & 0\end{array}\right]$, and $v_{i}=0$. One can note that

$$
\operatorname{curl}\left(g_{1}\right) \cdot g_{1}=-1 \neq 0, \quad \operatorname{curl}\left(g_{2}\right) \cdot g_{2}=0
$$

which, according to Remark 2 of Theorem 1, means that there exists an integrating factor for $c_{2}$ but not for $c_{1}$.

The codistribution $\Omega$ is by definition spanned by $\left\{g_{1}, g_{2}\right\}$ and it is easily verified that

$$
\Omega^{\perp}=\operatorname{span}\left\{\left[-A u_{1}, u_{2}, A u_{1} u_{2}-u_{2}\right]\right\}
$$

Since $\Omega^{\perp}$ is one dimensional it is involutive and therefore there exist two independent solutions of $d \lambda \Omega^{\perp}=$ 0 . The solutions can be determined by solving a set of ordinary differential equations (Isidori, 1995) and here the solutions are

$\lambda_{1}=(1-A)\left(u_{2}+u_{3}\right)-A u_{1} u_{2}+(2 A-1) u_{1}^{1 / A} u_{2}$

$\lambda_{2}=A u_{1}^{1 / A} u_{2}$
The integrating factors $\alpha_{i}$ can be calculated by solving the linear system of equations $d \lambda_{i}=\alpha_{i, 1} g_{1}+\alpha_{i, 2} g_{2}$ and the residual generators then become

$$
\begin{aligned}
\dot{x}_{i} & =-\beta x_{i}-\beta \lambda_{i}(u)+\alpha_{i, 1} v_{i}(u)+\alpha_{i, 2} v_{2}(u) \\
r_{i} & =x_{i}+\lambda_{i}(u)
\end{aligned}
$$

where $r_{i}$ are the residuals.

\section{FAULT DETECTABILITY}

To investigate fault detectability properties of the residual generators designed in the previous sections, assume that the original set of consistency relations have fault sensitivities according to

$$
c_{i}(\dot{u}, u)=s_{i}(f, u, \dot{u})
$$

where $f$ is a vector of faults. Recall Example 1 where the fault sensitivity of the consistency relation was given by $s_{i}=\theta_{2} f$.

The fault sensitivity of the residual generator (8) is given by

$$
\dot{r}+\beta r=\sum_{i=1}^{r} \alpha_{i}(u) s_{i}(f, u, \dot{u})
$$

This means that the residual $r$ will be sensitive to exactly those faults that affect the original consistency relations whose corresponding coefficient $\alpha_{i}(u) \not \equiv 0$. However, in operating points where $\alpha_{i}(u)$ is small or zero, fault sensitivity may be reduced or even removed. Thus, the coefficient $\alpha_{i}$ shapes the fault response in the residual. Note that the filtering imposed by the residual generator dynamics does not, except for the low pass filtering effect, remove fault sensitivity.

\section{HIGHER ORDER CONSISTENCY RELATIONS AND PARTIAL SOLUTIONS}

In previous sections, methodology to lower all first order derivatives in a consistency relation has been described. The topic of this section is to investigate what can be done if either we have a higher order consistency relation or if the conditions for the methodology to work are not satisfied. For the first case it will be shown how also higher order derivatives can be lowered one step and for the latter outline a procedure to lower some derivatives for the case when all derivatives can not be removed. It will also be clear that the two approaches can be combined in a straightforward manner.

When considering higher order derivatives, previous results directly applies. Let $u^{(k)}$ denote the k:th derivative of $u$. If the aim is to lower the derivatives $u_{i}^{\left(k_{i}\right)}$ one step, consider $z_{i}=u_{i}^{\left(k_{i}-1\right)}$ as variables. All other signals, except $\dot{z}_{i}=u_{i}^{\left(k_{i}\right)}$, are considered parameters. 
The procedure is best illustrated below by a small example.

Example 4. Consider the second order consistency relation below

$$
c(u, \dot{u}, \ddot{u})=u_{2} \ddot{u}_{1}+2 u_{1} \dot{u}_{2}
$$

The aim is to lower the second order derivative $\ddot{u}_{1}$ to a first order derivative and also to remove the derivative of $u_{2}$. Following the discussion above, consider $\dot{u}_{1}$ and $u_{2}$ as variables, and all lower order derivatives as parameters:

$$
A:=2 u_{1}, \quad z_{1}:=\dot{u}_{1}, \quad z_{2}:=u_{2}
$$

In these new variables, the consistency relation becomes

$$
c(z, \dot{z})=z_{2} \dot{z}_{1}+A \dot{z}_{2}
$$

Following the design procedure from previous sections, we can compute a potential $\lambda$ and an integrating factor $\alpha$ to obtain a new consistency relation $c^{\prime}=\alpha c$ as

$$
c^{\prime}=\alpha c=\frac{d}{d t}\left(\dot{u}_{1}+2 u_{1} \ln u_{2}\right)-2 \dot{u}_{1} \ln u_{2}
$$

By adding linear first order dynamics, a residual can then be computed where the only derivative needed is $\dot{u}_{1}$ instead of $\ddot{u}_{1}$ and $\dot{u}_{2}$.

Using the same approach, considering some signals as variables and some as parameters, it is sometimes possible to lower some of the derivatives in case it is not possible to lower all. This is illustrated in the following example.

Example 5. Consider the consistency relation

$$
c(u, \dot{u})=u_{2} \dot{u}_{1}+u_{1} u_{3} \dot{u}_{2}+\dot{u}_{3}^{2}
$$

which clearly does not fulfill the conditions in Theorem 1. In particular it is not on the form (5) because of the term $\dot{u}_{3}^{2}$. Consider only the first two terms in the consistency relation and assume $u_{3}$ to be a parameter $A$. This implies that the first two terms are considered to be on the form $u_{2} \dot{u}_{1}+A u_{1} \dot{u}_{2}$, which can be shown to satisfy the conditions in Theorem 1 . Following the methodology we obtain

$$
\lambda=u_{1} u_{2}^{u_{3}}, \quad \alpha=u_{2}^{u_{3}-1}
$$

and the resulting, transformed, consistency relation is

$$
c^{\prime}=\alpha c=\dot{\lambda}+u_{2}^{u_{3}-1} \dot{u}_{3}^{2}-u_{1} u_{2}^{u_{3}} \ln u_{2} \dot{u}_{3}
$$

where $\dot{u}_{1}$ and $\dot{u}_{2}$ has been removed while $\dot{u}_{3}$ remains. Note that the last term $u_{1} u_{2}^{u_{3}} \ln u_{2} \dot{u}_{3}$, which includes $\dot{u}_{3}$, appears due to that $u_{3}$ is a parameter in the design. This means that there exists a trade-off between lowering the derivative order of signals considered as variables and a possible raise of derivative order among signals considered as parameters.

\section{CONCLUSIONS}

The main results of this paper show how to lower, or if possible avoid, the need to estimate derivatives of known signals in order to compute a residual based on non-linear consistency relations. This is achieved by rewriting the problem into an integrability problem using state-space realization theory. An advantage of the approach, compared to for example the observer based approaches in (Persis and Isidori, 2001), is that general differential algebraic system descriptions can be handled in the same way as for example ordinary differential equations.

A key result is given by Theorem 1 which provides the designer with necessary and sufficient, easily verified, conditions to remove all derivatives in first order consistency relations. An attractive property of the method is that it is always possible to choose linear residual generator dynamics and stability of the residual is therefore easy to guarantee. For cases where it is not possible to remove all derivatives, the method can still be useful. Procedures are presented where the results can be used to provide partial solutions, e.g. lower some of the derivatives instead of all.

\section{REFERENCES}

Choquet-Bruhat, Y., C. DeWitt-Morette and M. Dillard-Bleick (1982). Analysis, manifolds and physics. revised ed.. North-Holland Publishing Co.. Amsterdam.

Delaleau, E. and W. Respondek (1995). Lowering the orders of derivatives of controls in generalized state space systems. Journal of Mathematical Systems, Estimation, and Control 5(3), 1-27.

Frisk, E. and M. Nyberg (2001). A minimal polynomial basis solution to residual generation for fault diagnosis in linear systems. Automatica 37, 1417-1424.

Gertler, J. (1991). Analytical redundancy methods in fault detection and isolation; survey and synthesis. Safeprocess. Baden-Baden, Germany. pp. 921.

Isidori, A. (1995). Nonlinear Control Systems. 3rd ed.. Springer Verlag.

Persis, C. De and A. Isidori (2001). A geometric approach to nonlinear fault detection and isolation. IEEE Automatic Control 46(6), 853-865.

Staroswiecki, M. and G. Comtet-Varga (2001). Analytical redundancy relations for fault detection and isolation in algebraic dynamic systems. $A u$ tomatica 37(5), 687-699. 